\title{
Optimization of ethylene glycol production from (D)-xylose via a synthetic pathway implemented in Escherichia coli
}

\author{
Ceren Alkim ${ }^{1,2,3,4^{*}+}$, Yvan Cam ${ }^{1,2,3,4 \dagger}$, Debora Trichez ${ }^{1,2,3}$, Clément Auriol 1,2,3,4 Lucie Spina $^{1,2,3}$, Amélie Vax ${ }^{1,2,3}$, \\ François Bartolo ${ }^{5}$, Philippe Besse ${ }^{5}$, Jean Marie François ${ }^{1,2,3,4}$ and Thomas Walther ${ }^{1,2,3,4^{*}}$
}

\begin{abstract}
Background: Ethylene glycol (EG) is a bulk chemical that is mainly used as an anti-freezing agent and a raw material in the synthesis of plastics. Production of commercial EG currently exclusively relies on chemical synthesis using fossil resources. Biochemical production of ethylene glycol from renewable resources may be more sustainable.

Results: Herein, a synthetic pathway is described that produces EG in Escherichia coli through the action of (D)xylose isomerase, (D)-xylulose-1-kinase, (D)-xylulose-1-phosphate aldolase, and glycolaldehyde reductase. These reactions were successively catalyzed by the endogenous xylose isomerase (Xy|A), the heterologously expressed human hexokinase (Khk-C) and aldolase (Aldo-B), and an endogenous glycolaldehyde reductase activity, respectively, which we showed to be encoded by yqhD. The production strain was optimized by deleting the genes encoding for (D)-xylulose-5 kinase (xy/B) and glycolaldehyde dehydrogenase (aldA), and by overexpressing the candidate glycolaldehyde reductases YqhD, GldA, and FucO. The strain overproducing FucO was the best EG producer reaching a molar yield of 0.94 in shake flasks, and accumulating $20 \mathrm{~g} / \mathrm{L} \mathrm{EG}$ with a molar yield and productivity of $0.91 \mathrm{and} 0.37 \mathrm{~g} /(\mathrm{L} . \mathrm{h})$, respectively, in a controlled bioreactor under aerobic conditions.
\end{abstract}

Conclusions: We have demonstrated the feasibility to produce EG from (D)-xylose via a synthetic pathway in E. coli at approximately $90 \%$ of the theoretical yield.

Keywords: Synthetic metabolic pathway, Ethylene glycol, Xylose, Metabolic engineering, Escherichia coli

\section{Background}

Ethylene glycol (EG; 1,2-ethanediol) and its polymers are used in an expanding range of products that include heat transfer fluids, lubricants, surfactants, explosives, cosmetics and plastics [1-3]. The global demand of EG was approximately 21 million tons in 2010 and it is expected to be more than 28 million tons per year by 2015 [4]. Currently, industrial production of EG exclusively relies on chemical synthesis which proceeds through steam cracking of petrol to obtain ethylene, and the oxidation of ethylene to ethylene oxide which is followed by thermal

\footnotetext{
*Correspondence: alkim@insa-toulouse.fr; thomas.walther@ insa-toulouse.fr

${ }^{\dagger}$ Ceren Alkim and Yvan Cam contributed equally to this work

${ }^{4}$ TWB, 3 rue Ariane, 31520 Ramonville-St. Agnes, France

Full list of author information is available at the end of the article
}

hydrolysis to yield EG [5]. The decreasing availability of petrol and increasing market prices have prompted the search for biochemical production processes to synthesize value-added chemicals from renewable resources $[6,7]$. In this context, the microbial production of EG receives increasing attention, and the present study investigates the potential to apply a previously developed synthetic pathway for xylose assimilation [8] for the production of EG.

Three different strategies for the biochemical production of EG from (D)-xylose using engineered Escherichia coli strains have recently been described by Liu et al. [9], Stephanopoulos et al. [10], and our group [8] (Table 1), all of which provide access to EG at a theoretical maximum yield of $1 \mathrm{~mol} / \mathrm{mol}$. The pathway developed by Liu et al. [9] (in the following termed xylonate 
pathway) proceeds via the oxidation of (D)-xylose to (D)-xylonate, the dehydration of (D)-xylonate to produce 2-dehydro-3-deoxy-D-pentonate, the aldolytic cleavage of 2-dehydro-3-deoxy-D-pentonate to form pyruvate and glycolaldehyde, and the reduction of the latter to obtain EG. An E. coli strain which was deleted in (D)-xylose isomerase, encoded by $x y l A$, and which expressed this pathway produced $11.7 \mathrm{~g} / \mathrm{L} \mathrm{EG}$ at a yield of $0.29 \mathrm{~g} \mathrm{EG}$ per gram (D)-xylose $(0.71 \mathrm{~mol} / \mathrm{mol})$ in a controlled bioreactor (Table 1). Stephanopoulos et al. [10] developed a pathway [in the following termed (D)-ribulose-1P pathway] that converts (D)-xylose into (D)-ribulose-1P via the sequential action of (D)-xylose isomerase, (D)-xylulose epimerase, and (D)-ribulose-1 kinase. (D)-ribulose$1 \mathrm{P}$ is then cleaved aldolytically into dihydroxyacetone phosphate (DHAP) and glycolaldehyde before the latter is reduced to yield EG. A genetically engineered E. coli strain expressing this pathway produced $3.5 \mathrm{~g} / \mathrm{L}$ of EG in shake flasks with a yield of $0.35 \mathrm{~g}$ EG per gram xylose consumed $(0.84 \mathrm{~mol} / \mathrm{mol}$, Table 1$)$. In a controlled bioreactor the strain produced $42 \mathrm{~g} / \mathrm{L}$ ethylene glycol from an unknown amount of (D)-xylose [10].

We recently developed a synthetic pathway [Fig. 1, in the following termed (D)-xylulose-1P pathway] that can transform (D)-xylose into EG and glycolic acid (GA) [8] by proceeding via the isomerization of (D)-xylose to (D)xylulose, phosphorylation of (D)-xylulose to obtain (D)xylulose-1P, aldolytic cleavage of (D)-xylulose-1P to form DHAP and glycolaldehyde, and reduction or oxidation of glycolaldehyde to yield EG or GA, respectively. Growth of a strain deleted in the xylulose-5 kinase encoding $x y l B$ gene on (D)-xylose was restored when (D)-xylulose-1 kinase and (D)-xylulose-1P aldolase, provided by the human genes encoding ketohexokinase- $C$ and aldolase- $B$ enzymes were expressed simultaneously. This strain was then further optimized and eventually produced GA at a molar yield of 0.9 [8].

We herein investigated the potential of our synthetic pathway to produce EG at high yield. We identified the major glycolaldehyde reductase in E.coli, and optimized the strain by deleting the endogenous glycolaldehyde oxidase and overexpressing different candidate glycolaldehyde reductases that varied in regard to their cofactor specificities. We then analyzed the impact of different aeration conditions on the production of EG, and found that fully aerobic conditions were preferable over oxygenlimiting and anaerobic conditions. This strategy led us to obtain an engineered strain that produced $20 \mathrm{~g} / \mathrm{L}$ EG at a molar yield and productivity of approximately 0.9 and $0.3 \mathrm{~g} /(\mathrm{L} \mathrm{h})$, respectively, in a controlled bioreactor.

\section{Results and discussion}

The aldehyde reductase YqhD is the major glycolaldehyde reductase in $E$. coli

We have recently shown that an $E$. coli strain deleted in $x y l B$ and expressing the synthetic pathway enzymes xylulose-1 kinase and xylulose-1P aldolase, provided by expression of human $k h k-C$ and aldo $B$ genes, respectively, was able to grow on xylose [8]. The strain produced EG at a yield of $0.19 \mathrm{~g} / \mathrm{g}(0.45 \mathrm{~mol} / \mathrm{mol}$, Table 1$)$. To optimize this strain for EG production we first set out to identify the natural glycolaldehyde reductase in E. coli. It was earlier shown that the aldehyde reductases encoded by $d k g A(y q h E), d k g B(y a f B), y q h D, y e a E$, and

Table 1 Ethylene glycol production with different engineered $E$. coli strains expressing the synthetic pathway

\begin{tabular}{|c|c|c|c|c|c|}
\hline \multirow{2}{*}{$\begin{array}{l}\text { Experimental conditions } \\
\text { Xylonate pathway expressed in E. coli } \Delta x y / A \text { mutant }\end{array}$} & \multirow[t]{2}{*}{$\begin{array}{l}\text { Final conc. } \\
(\mathrm{g} / \mathrm{L})\end{array}$} & \multicolumn{2}{|c|}{$\begin{array}{l}\text { Yield }^{\mathrm{a}} \\
(\mathrm{mol} / \mathrm{mol})(\mathrm{g} / \mathrm{g})\end{array}$} & \multirow[t]{2}{*}{$\begin{array}{l}\text { Productivity } \\
{[\mathrm{g} /(\mathrm{I} h)]}\end{array}$} & \multirow{2}{*}{$\begin{array}{l}\text { References } \\
{[9]}\end{array}$} \\
\hline & & & & & \\
\hline $\begin{array}{l}\text { Shake flask }(\mathrm{MM}+4 \mathrm{~g} / \mathrm{L} \text { xylose }+1 \mathrm{~g} / \mathrm{L} \text { pep- } \\
\text { tone }+0.5 \mathrm{~g} / \mathrm{L} \text { yeast extract })\end{array}$ & 0.92 & 0.55 & 0.23 & ni & \\
\hline $\begin{array}{l}\text { Bioreactor (MM }+40 \mathrm{~g} / \mathrm{L} \text { xylose }+10 \mathrm{~g} / \mathrm{L} \text { pep- } \\
\text { tone }+5 \mathrm{~g} / \mathrm{L} \text { yeast extract, batch process })\end{array}$ & 11.7 & 0.71 & 0.29 & 0.24 & \\
\hline \multicolumn{5}{|c|}{ (D)-ribulose-1P pathway (dte + fucA + fucO + fuck) expressed in E. coli $\Delta x y l B \Delta$ aldA ( $\Delta$ endA $\Delta$ rec $A)$ mutant } & {$[10]$} \\
\hline Shake flask (MM + $10 \mathrm{~g} / \mathrm{L}$ xylose) & 3.5 & 0.84 & 0.35 & ni & \\
\hline $\begin{array}{l}\text { Bioreactor (MM + unknown amount of xylose, fed-batch } \\
\text { process) }\end{array}$ & 42.0 & ni & ni & 0.6 & \\
\hline \multicolumn{5}{|c|}{ (D)-xylulose-1P pathway $(k h k C+$ aldoB) expressed in E. coli $\Delta x y / B$ mutant } & {$[8]$} \\
\hline Shake flask (MM + $10 \mathrm{~g} / \mathrm{L}$ xylose) & 1.9 & 0.45 & 0.19 & ni & \\
\hline \multicolumn{5}{|c|}{ (D)-xylulose-1P pathway ( $k h k C+$ aldoB + fucO) expressed in E. coli $\Delta x y l B \Delta$ aldA mutant } & This study \\
\hline Shake flask (MM + $10 \mathrm{~g} / \mathrm{L}$ xylose) & 4.1 & 0.94 & 0.39 & ni & \\
\hline $\begin{array}{l}\text { Bioreactor (MM }+55 \mathrm{~g} / \mathrm{L} \text { xylose }+1 \mathrm{~g} / \mathrm{L} \text { peptone, batch } \\
\text { process) }\end{array}$ & 20.3 & 0.91 & 0.38 & 0.37 & \\
\hline
\end{tabular}




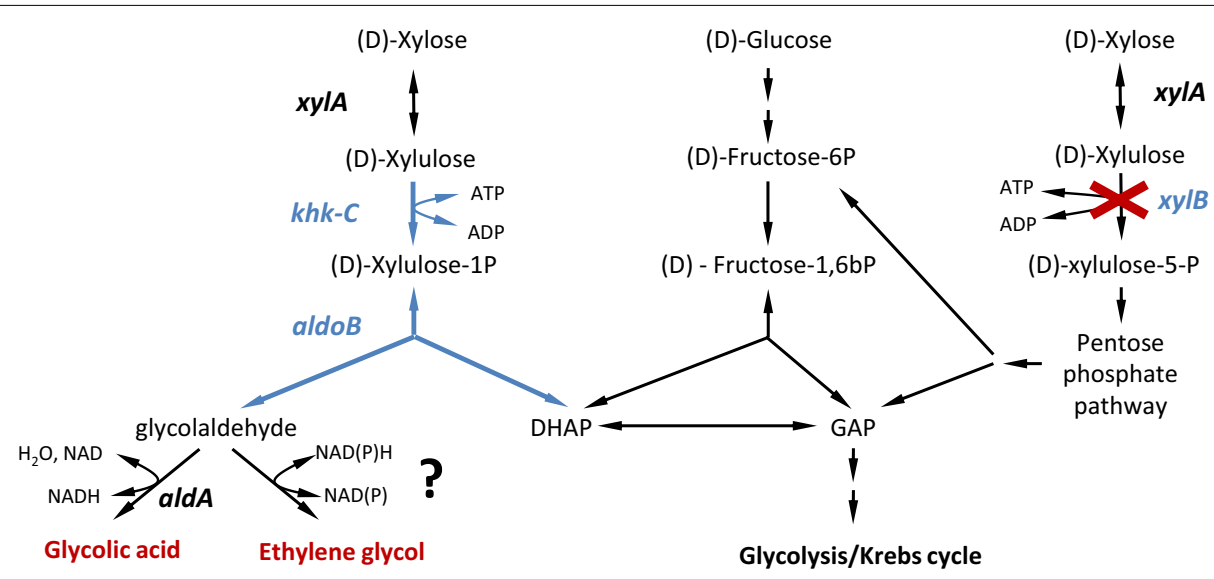

Fig. 1 Synthetic (b/ue) and natural (black) D-xylose assimilation pathways. In the synthetic pathway (D)-xylose is transformed to (D)-xylulose by endogenous xylose isomerase (Xy|A). (D)-xylulose-1-kinase (Khk-C) phosphorylates (D)-xylulose to obtain (D)-xylulose-1P, and (D)-xylulose-1-phosphate aldolase (Aldo-B) cleaves (D)-xylulose-1P into glycolaldehyde and DHAP. Ethylene glycol is produced via the action of an unknown endogenous aldehyde reductase.

$y g h Z$ have glycolaldehyde reductase activity [11]. In addition, the L-1,2-propanediol oxidoreductase encoded by $f u c O$ [12-14], and the glycerol dehydrogenase encoded by gldA [15] were reported to reduce glycolaldehyde [16, 17]. We therefore tested the impact of deleting these enzymes individually or in combination on the capability of E. coli strains to reduce glycolaldehyde to EG. The strains carrying the desired mutations were cultivated in shake flasks on minimal medium containing (D)-xylose as the only carbon source. Glycolaldehyde was added at a concentration of $10 \mathrm{mM}$ in the early exponential growth phase (at OD $\sim 1$ ) and the accumulation of EG was measured by HPLC after $10 \mathrm{~h}$ of incubation (Fig. 2a). The wild-type strain produced $3.3 \mathrm{mM}$ EG under these conditions. We found that only the deletion of $y q h D$ significantly decreased the conversion of glycolaldehyde to EG to approximately $30 \%$ of the wild-type levels. No further decrease of EG production could be observed after additional deletion of $f u c O$ and $g l d A$, which had been employed as glycolaldehyde reductases elsewhere [16, 17] (Fig. 2a). Our results indicate that YqhD functions as the major glycolaldehyde reductase in E. coli. This result is somewhat surprising when regarding the comparatively high $\mathrm{K}_{\mathrm{m}}$ value $(28 \mathrm{mM})$ of YqhD for glycolaldehyde [11]. It is, however, in agreement with the general role of YqhD as a promiscuous aldehyde reductase and detoxifying enzyme in E. coli [18]. Moreover, our data indicates that there is at least one additional enzyme capable of reducing glycolaldehyde in the absence of $y q h D$, but we did not identify this enzyme in our study.

We then investigated the transcriptional regulation of the above listed aldehyde reductases in strain
Pen205 ( $\triangle x y l B$ mutant expressing the synthetic pathway enzymes Khk-C and Aldo-B), and in wild-type cells exposed to $10 \mathrm{mM}$ glycolaldehyde. We re-analyzed our recently published data on the genome-wide transcriptional activity under these conditions [11] and found that among the above listed eight aldehyde reductase-encoding genes only $y q h D$ and $d k g A$ were induced in strain Pen205 and in the presence of glycolaldehyde (Fig. 2b; Additional file 1: Table S1). The $d k g A$ gene was eight- and tenfold induced in strain Pen205 and in wild type incubated with $10 \mathrm{mM}$ glycolaldehyde, respectively, whereas $y q h D$ showed the strongest transcriptional upregulation of $~ 20$-fold in strain Pen205 and 26-fold in the presence of glycolaldehyde. The induction of both $y q h D$ and $d k g A$ could be explained by their regulation via the same transcriptional regulator, YqhC [19], whose expression was increased by sevenfold in strain Pen205 and 15-fold in wild types strain incubated glycolaldehyde.

We then analyzed the impact of deleting $y q h D$ on growth and EG production of cells expressing the synthetic pathway. Strain Pen334 ( $\Delta x y l B \Delta y q h D$ double mutant expressing the synthetic pathway) was incubated in shake flasks on mineral medium containing (D)-xylose as the sole carbon source. Growth and products formation were monitored by measuring $\mathrm{OD}_{600}$ and by carrying out HPLC analyses, respectively. We found that only very small amounts of EG $(2.93 \pm 0.23 \mathrm{mM})$ were produced by the $y q h D$-deleted strain Pen334, whereas strain Pen205 accumulated $31 \mathrm{mM}$ EG (Fig. 3). In addition, strain Pen334 consumed only half of the $70 \mathrm{mM}$ (D)-xylose that were initially present in the medium and was unable to sustain growth above 4 units $\mathrm{OD}_{600}$, which suggests that 


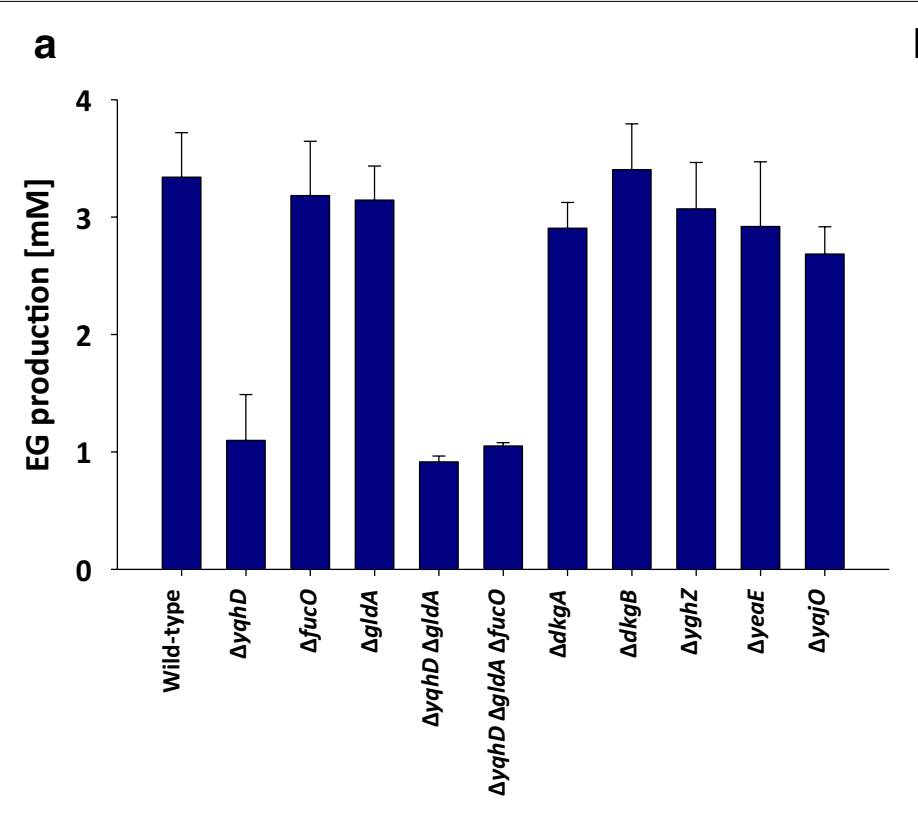

b

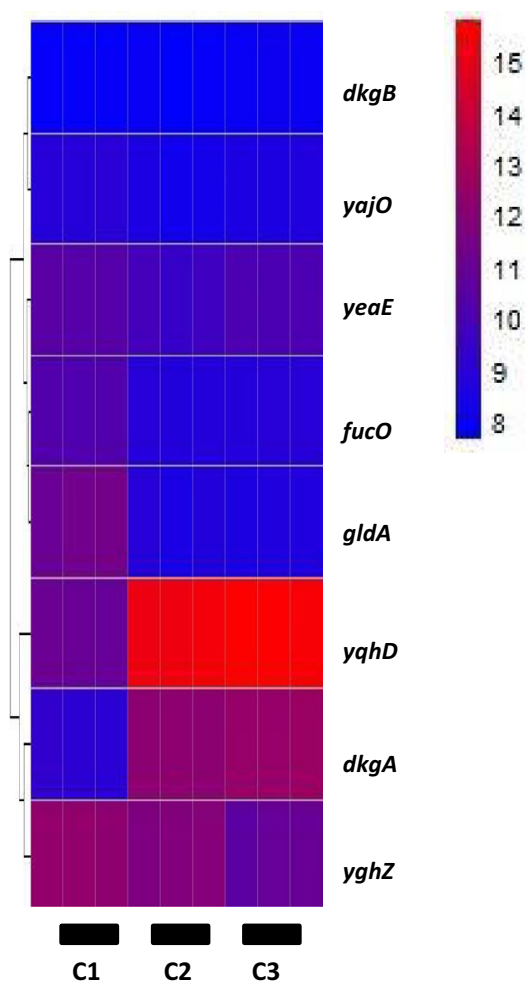

Fig. 2 Identification of YahD as the major glycolaldehyde reductase in E. coli. a Production of ethylene glycol by E. coli strains depending on the deletion of candidate glycolaldehyde reductases. Cells were cultivated on mineral (D)-xylose medium and exposed to $10 \mathrm{mM}$ glycolaldehyde. Production of ethylene glycol was estimated after $10 \mathrm{~h}$ of incubation. b Log2 transformed expression levels of candidate glycolaldehyde reductases in wild-type cells (C1), strain Pen205 ( $\Delta x y \mid B$ expressing pEXT20-khk-C-aldoB) (C2), and wild-type cells exposed to 10 mM glycolaldehyde (C3). Genes were clustered according to the Euclidean distance between their expression levels using complete-linkage clustering [30]. Red and blue correspond to high and low expression levels, respectively, using arbitrary units.

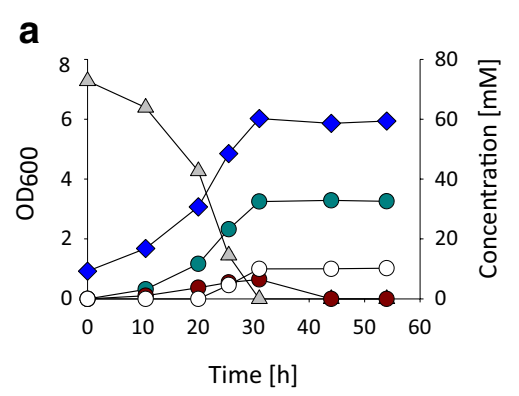

b

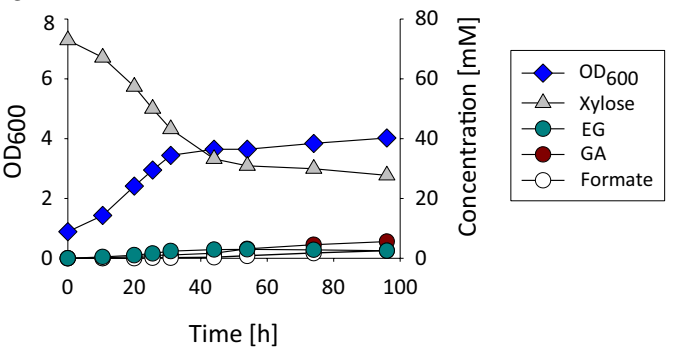

Fig. 3 Growth and product formation kinetics depending on the presence and absence of YqhD. a Pen205 ( $\Delta x y / B$ expressing pEXT20-khk-C-aldoB) and b Pen334 ( $\Delta x y / B \Delta y q h D$ pEXT20-khk-C-aldoB) were cultivated on mineral medium containing (D)-xylose at $70 \mathrm{mmol} / \mathrm{L}$ initial concentration. Experiments were performed in $250 \mathrm{~mL}$ shake flask containing $50 \mathrm{~mL}$ medium.

the deletion of $y q h D$ had caused the accumulation of toxic levels of glycolaldehyde or a derivative of this compound. Taken together, these data indicate that YqhD is the major glycolaldehyde reductase in $E$. coli, and suggest that this enzyme is induced in the presence of the synthetic pathway to detoxify glycolaldehyde to EG.

\section{Metabolic engineering to increase production of ethylene} glycol

As indicated in Table 2, the sole expression of Khk-C and Aldo-B in the wild-type E. coli strain (Pen877) did not allow the production EG or GA (Table 2). This result suggested that our synthetic pathway was outcompeted 
Table 2 Product yields $(Y)$ of engineered E. coli strains expressing the synthetic pathway

\begin{tabular}{|c|c|c|c|c|c|c|c|}
\hline Strain name & Deletions & Plasmids & $Y_{\text {Biomass }}(g / g)$ & $\mathrm{Y}_{\mathrm{EG}}(\mathrm{mol} / \mathrm{mol})$ & $\mathrm{Y}_{\mathrm{GA}}(\mathrm{mol} / \mathrm{mol})$ & $\mathrm{Y}_{\text {Acetate }}(\mathrm{mol} / \mathrm{mol})$ & $Y_{\text {Formate }}(\mathrm{mol} / \mathrm{mol})$ \\
\hline Pen877 & & pEXT20-khkC-aldoB & $0.11 \pm 0.00$ & $0.00 \pm 0.00$ & $0.00 \pm 0.00$ & $1.07 \pm 0.02$ & $0.20 \pm 0.01$ \\
\hline Pen205 & $\Delta x y \mid B$ & pEXT20-khkC-aldoB & $0.14 \pm 0.00$ & $0.45 \pm 0.01$ & $0.09 \pm 0.00$ & $0.00 \pm 0.00$ & $0.14 \pm 0.01$ \\
\hline Pen885 & $\Delta x y \mid B$ & $\begin{array}{l}\text { pEXT20-khkC- } \\
\text { aldoB + pACT3-empty }\end{array}$ & $0.14 \pm 0.00$ & $0.33 \pm 0.01$ & $0.00 \pm 0.00$ & $0.00 \pm 0.00$ & $0.17 \pm 0.01$ \\
\hline Pen222 & $\Delta x y \mid B$ & $\begin{array}{l}\text { pEXT20-khkC- } \\
\text { aldoB + pACT3-gldA }\end{array}$ & $0.15 \pm 0.01$ & $0.37 \pm 0.00$ & $0.00 \pm 0.00$ & $0.01 \pm 0.00$ & $0.13 \pm 0.03$ \\
\hline Pen223 & $\Delta x y \mid B$ & $\begin{array}{l}\text { pEXT20-khkC- } \\
\text { aldoB + pACT3-yqhD }\end{array}$ & $0.18 \pm 0.03$ & $0.33 \pm 0.00$ & $0.03 \pm 0.03$ & $0.00 \pm 0.00$ & $0.22 \pm 0.00$ \\
\hline Pen644 & $\Delta x y \mid B$ & $\begin{array}{l}\text { pEXT20-khkC- } \\
\text { aldoB + pACT3-fucO }\end{array}$ & $0.14 \pm 0.01$ & $0.36 \pm 0.01$ & $0.06 \pm 0.00$ & $0.01 \pm 0.00$ & $0.18 \pm 0.00$ \\
\hline Pen334 & $\Delta x y \mid B \Delta y q h D$ & pEXT20-khkC-aldoB & $0.14 \pm 0.00$ & $0.07 \pm 0.01$ & $0.04 \pm 0.00$ & $0.02 \pm 0.00$ & $0.01 \pm 0.00$ \\
\hline Pen325 & $\Delta x y \mid B \Delta a l d A$ & pEXT20-khkC-aldoB & $0.12 \pm 0.01$ & $0.88 \pm 0.00$ & $0.00 \pm 0.00$ & $0.00 \pm 0.00$ & $0.02 \pm 0.02$ \\
\hline Pen361 & $\Delta x y \mid B \Delta a l d A \Delta g l c D$ & pEXT20-khkC-aldoB & $0.10 \pm 0.00$ & $0.87 \pm 0.00$ & $0.02 \pm 0.00$ & $0.00 \pm 0.00$ & $0.02 \pm 0.00$ \\
\hline Pen332 & $\Delta x y \mid B \Delta a l d A$ & $\begin{array}{l}\text { pEXT20-khkC- } \\
\text { aldoB + pACT3-gldA }\end{array}$ & $0.08 \pm 0.00$ & $0.52 \pm 0.00$ & $0.00 \pm 0.00$ & $0.00 \pm 0.00$ & $0.00 \pm 0.00$ \\
\hline Pen333 & $\Delta x y \mid B \Delta a l d A$ & $\begin{array}{l}\text { pEXT20-khkC- } \\
\text { aldoB + pACT3-yqhD }\end{array}$ & $0.11 \pm 0.01$ & $0.90 \pm 0.00$ & $0.00 \pm 0.00$ & $0.00 \pm 0.01$ & $0.01 \pm 0.00$ \\
\hline Pen641 & $\Delta x y \mid B \Delta a l d A$ & $\begin{array}{l}\text { pEXT20-khkC- } \\
\text { aldoB + pACT3-fucO }\end{array}$ & $0.16 \pm 0.01$ & $0.94 \pm 0.00$ & $0.00 \pm 0.00$ & $0.00 \pm 0.01$ & $0.07 \pm 0.01$ \\
\hline
\end{tabular}

Data is presented as means \pm standard deviations of at least two independent experiments. For strains Pen334 and 332 only $\sim 50 \%$ of the initially present (D)-xylose were consumed. All experiments were performed in $250 \mathrm{~mL}$ shake flasks filled with $50 \mathrm{~mL}$ medium and shaken at $200 \mathrm{rpm}$.

by the natural pathway which channels xylose-derived carbon through the pentose phosphate pathway. Consistently, we showed that the deletion of the xylulose- 5 kinase encoded by $x y l B$ (strain Pen205) was found to be mandatory to enable significant carbon flow and thus production of EG and GA through the synthetic pathway.

We next tried to increase EG production by overexpressing the major glycolaldehyde reductase YqhD. The gene was cloned into the medium-copy plasmid pACT3 and co-expressed with the synthetic pathway in a $\triangle x y l B$ mutant strain (Pen223). Contrary to expectation, we found that the overproduction of this enzyme caused a drop of the EG yield from 0.45 to $0.33 \mathrm{~mol} / \mathrm{mol}$ (Table 2). Since an almost identical decrease in EG production was observed for strain Pen 885 that carried the empty pACT3 plasmid (Table 2), we concluded that the slight decrease in EG production was likely due the increased metabolic burden caused by the additional plasmid. We then hypothesized that the overexpression of $y q h D$ had no positive effect because cofactor supply for this NADPHdependent enzyme had been limited. We therefore tested the impact of expressing the NADH-dependent enzymes FucO and GldA which were previously shown to have glycolaldehyde reductase activity [16, 17]. As shown in Table 2, the expression of those enzymes also resulted in a decrease of EG production that was comparable to what had been observed for the overproduction of YqhD.
These results suggested that the glycolaldehyde reductases were outcompeted by the glycolaldehyde dehydrogenase(s) which oxidizes glycolaldehyde to glycolate (Fig. 1). We have previously shown that overexpression of the short chain aldehyde dehydrogenase, AldA [20] abolished EG production in a strain that expressed our synthetic pathway [8]. We therefore tested the effect of deleting this gene on EG production. We found that the aldA-deleted strain (Pen325) produced EG at a molar yield of 0.88 which corresponded to an increase of nearly $100 \%$ compared to strain Pen205. This result showed that AldA plays a major role in the assimilation of the glycolaldehyde that is released by our synthetic pathway. It is of interest to note that the observed positive effect of deleting aldA is at variance with data of Liu et al. [9] who found that the aldA deletion was toxic for the cells. It, however, agrees with Stephanopoulos et al. [10] who proposed to delete aldA to increased EG production.

The theoretical C2-compound yield $\left(\mathrm{Y}_{\mathrm{C} 2}=([\mathrm{GA}]\right.$ $+[E G]) /\left[\right.$ xylose $\left.\left._{\text {consumed }}\right]\right)$ of our pathway is $1 \mathrm{~mol}$ $([\mathrm{EG}]+[\mathrm{GA}])$ per mol xylose (Fig. 1). Thus, despite the increase of EG production to $88 \%$ of the theoretical yield in strain Pen325, part of the glycolaldehyde was still consumed by another pathway. We therefore tested whether an additional glycolaldehyde dehydrogenase was active in our strains which could explain the incomplete recovery of the glycolaldehyde-derived carbon fraction. The inactivation of glycolate oxidase, encoded by glcDEFG [21], 
results in the incapacity of E. coli to consume GA [11, 22]. We therefore deleted the glycolate oxidase subunit $g l c D$ in addition to ald $A$ to obtain strain Pen361 ( $\triangle x y l B \triangle$ ald $A$

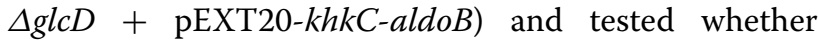
we could detect GA in the supernatant of this strain which would be an indicator for the presence of another unknown glycolaldehyde dehydrogenase. We observed that strain Pen361 produced only trace amounts of GA (0.02 $\mathrm{mol} / \mathrm{mol}$, Table 2). This result showed that the apparent $12 \%$ loss of glycolaldehyde observed for strain Pen325 did not occur through the glycolate/glyoxylate pathway. We therefore conclude that a small fraction of glycolaldehyde was consumed by an unknown metabolic pathway which remains to be identified.

A further incremental improvement of EG production to molar yields of 0.9 and 0.94 was achieved when the glycolaldehyde reductases YqhD or FucO, respectively, were overexpressed in the strain carrying the aldA deletion. In contrast to the positive impact of YqhD and FucO, the overexpression of GldA only had a slightly negative effect (Table 2). YqhD and FucO were earlier applied as glycolaldehyde reductases $[9,10]$. We here compared the effect of expressing either of these enzymes and found that both had a small positive effect on EG production.

\section{Optimization of aeration conditions to increase production of ethylene glycol}

After showing that the deletion of aldA encoding the glycolaldehyde dehydrogenase and the overexpression of $y q h D$ or $f u c O$ encoding glycolaldehyde reductases increased the production of EG, we investigated which aeration conditions are best for EG production by our synthetic pathway. We hypothesized that decreasing aeration of the cultures may increase the supply of reducing cofactors in particular for the NADH-dependent glycolaldehyde reductases GldA and FucO.

We varied the oxygen supply to our cultures by incubating them in $250 \mathrm{~mL}$ shake flasks which were filled with $50 \mathrm{~mL}$ (condition 1, as above) or $100 \mathrm{~mL}$ (condition 2) of culture medium, thus imposing gradually decreasing aeration with increasing medium volume [23]. In addition, anaerobic cultivation conditions (condition 3) were analyzed by cultivating the cells in completely filled $100 \mathrm{~mL}$ glass bottles that were sealed with a rubber cap. The anaerobic bottles were inoculated aerobically and anaerobic conditions were attained after only few minutes of cultivation due the oxygen consumption of the growing culture. Absence of oxygen was verified by the characteristic pink color of the redox indicator resazurin [24].

We tested the impact of these different aeration conditions on $\triangle x y l B$ aald $A$ double mutant strain Pen325 which only expressed the synthetic pathway that comprised of Khk-C and Aldo-B, or the synthetic pathway in combination with one of the three glycolaldehyde reductases GldA (Pen332), YqhD (Pen333), or FucO (Pen641). The results are summarized in Table 3 . We found that fully aerobic conditions (condition 1) were preferable over micro-aerobic (condition 2) and anaerobic conditions (condition 3) for the production of EG when using the strains that overproduced YqhD (Pen333), FucO (Pen641), or which contained no additional glycolaldehyde reductase (Pen325). Upon decreasing oxygen supply and in the absence of oxygen these strains accumulated increasing amounts of acetate and succinate in the supernatant, which negatively affected biomass and EG yield. Curiously we could detect up to $0.17 \mathrm{~mol} / \mathrm{mol}$ formate (Pen641) in the supernatant of these cultures under micro-aerobic conditions but not under anaerobic conditions. A slight exception of this preference for fully aerobic conditions was strain Pen332 which expressed GldA. These cells exhibited a significantly increased production of EG of $0.74 \mathrm{~mol} / \mathrm{mol}$ under micro-aerobic conditions as compared to only $0.52 \mathrm{~mol} / \mathrm{mol}$ under fully aerobic conditions.

These results indicate that (1) fully aerobic conditions are required for the production of EG at high yield, and (2) further support the hypothesis that an unknown glycolaldehyde-consuming pathway must be present in $E$. coli, because the recovery of the $\mathrm{C} 2$ carbon fraction (in the form of EG or GA) dropped to only $\sim 0.5-0.6 \mathrm{~mol}$ per mol xylose under anaerobic conditions.

\section{Production of ethylene glycol in controlled bioreactors}

To explore the capacity of strain Pen641 for maximal EG to production, we analyzed growth and products formation of this strain in controlled bioreactors under two different aeration conditions. We imposed fully aerobic conditions in one experiment by maintaining the dissolved oxygen tension above $40 \%$ throughout the cultivation. In parallel, we analyzed the impact of a reduced oxygen supply by allowing the dissolved oxygen tension to decrease to $2 \%$ in another experiment. Strain Pen641 was pre-cultured in (D)-xylose mineral medium until cells grew exponentially. These cells were then used to inoculate $0.5 \mathrm{l}$ bioreactors that contained $250 \mathrm{~mL}$ mineral medium with an initial (D)-xylose concentration of $55 \mathrm{~g} / \mathrm{L}$ supplemented with $1 \mathrm{~g} / \mathrm{L}$ tryptone and $0.5 \mathrm{~g} / \mathrm{L}$ yeast extract at an OD of $\sim 1.2$. The $\mathrm{pH}$ of the cultures was kept at 7.0 by the addition of $10 \mathrm{M} \mathrm{KOH}$. The fermentation kinetics of these experiments are depicted in Fig. 4. Under aerobic conditions strain Pen641 produced $20 \mathrm{~g} / \mathrm{L}$ EG with a volumetric productivity of $0.37 \mathrm{~g} /(\mathrm{L} \mathrm{h})$. The molar EG yield on (D)-xylose was $0.9(0.38 \mathrm{~g} / \mathrm{g})$ and only small amounts of the metabolic by-products glycolaldehyde $(1 \mathrm{~g} / \mathrm{L})$ and formate $(0.45 \mathrm{~g} / \mathrm{L})$ were produced (Fig. 4a). Under oxygen-limited conditions strain Pen641 
Table 3 The impact of variations in oxygen supply on the production of ethylene glycol

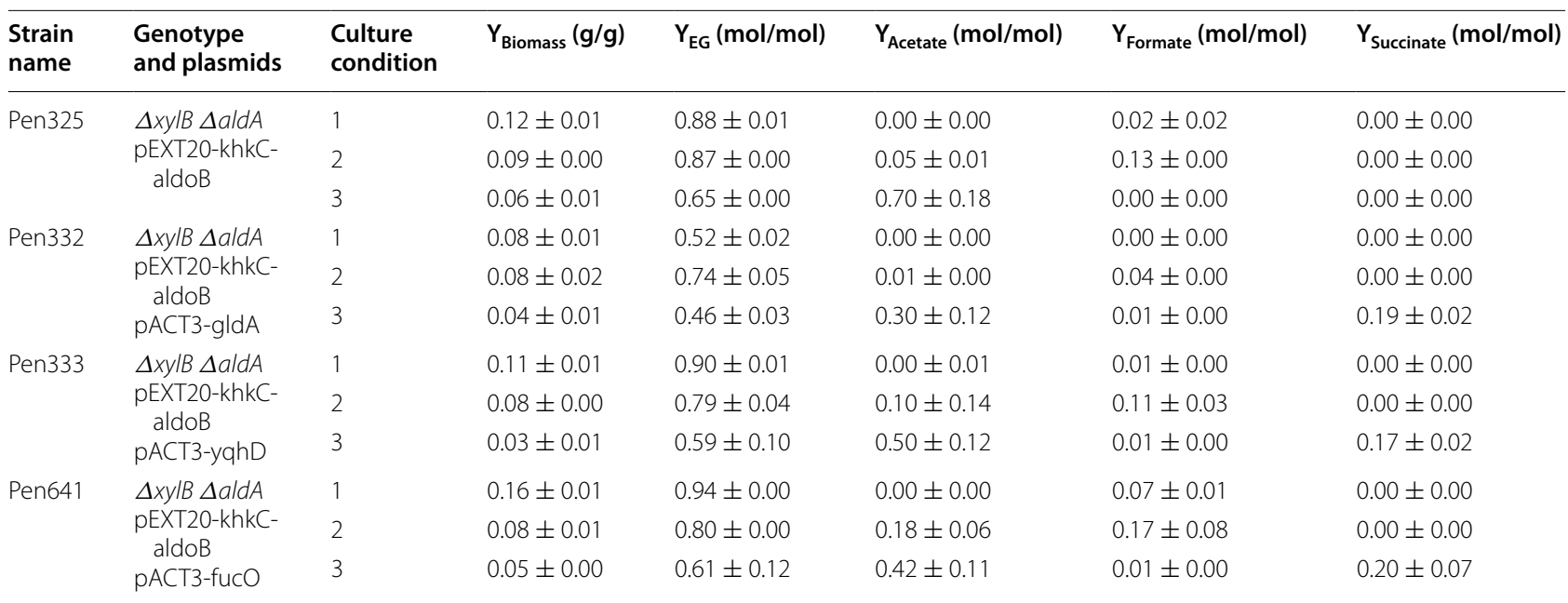

(1) $50 \mathrm{~mL}$ medium in $250 \mathrm{~mL}$ shake flasks, $200 \mathrm{rpm}$. (2) $100 \mathrm{~mL}$ medium in $250 \mathrm{~mL}$ shake flasks, $100 \mathrm{rpm}$. (3) anaerobic cultures, $200 \mathrm{rpm}$. Data is presented as means \pm standard deviations of at least two independent experiments.
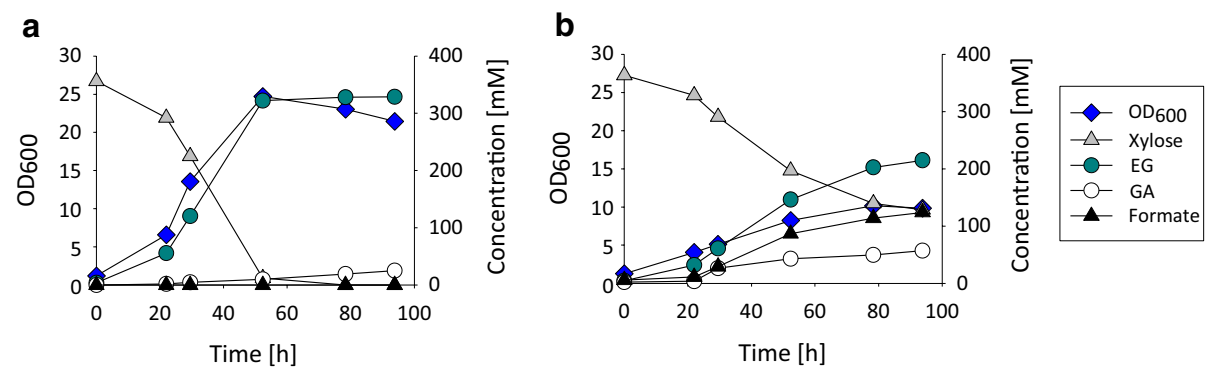

Fig. 4 Growth and product formation kinetics of strain Pen641 in controlled bioreactors. Cells were cultivated under fully aerobic conditions (a), or under micro-aerobic conditions (b) on xylose mineral medium enriched with $1 \mathrm{~g} / \mathrm{L}$ tryptone and $0.5 \mathrm{~g} / \mathrm{L}$ yeast extract. Initial (D)-xylose concentration was $55 \mathrm{~g} / \mathrm{L}$. Aerobic and micro-aerobic conditions were imposed by maintaining the dissolved oxygen tension above 40 and $2 \%$, respectively.

produced EG also at a molar yield of 0.9 . However, when oxygen supply was limited the strain was not able to consume all the xylose in the medium and accumulated only $13.3 \mathrm{~g} / \mathrm{L}$ EG with a significantly decreased volumetric productivity of only $0.15 \mathrm{~g} /(\mathrm{L} . \mathrm{h})$ (Fig. 4b). Under these conditions, cells exhibited a twofold reduced growth rate $(0.05 / \mathrm{h}$ as compared to $0.1 / \mathrm{h}$ under fully aerobic conditions), and they ceased growth at an $\mathrm{OD}_{600}$ of $\sim 10$ presumably because the acetate concentration of $7 \mathrm{~g} / \mathrm{L}$ in the medium became growth inhibiting. These data further support the notion that fully aerobic conditions are preferable for EG production when using the described synthetic pathway. The almost complete absence of metabolic by-products and notably acetate under these conditions suggest that it is possible to accumulate EG to significantly higher concentrations in fed-batch fermentation processes.

The EG yield and productivity achieved by our strain are by 27 and $54 \%$, respectively, higher than for the strain carrying the xylonate pathway engineered by Liu et al. [9]. Given the strong transient accumulation of glycolic acid in the fermentation experiment of Liu et al. [9], the increase of EG yield obtained with our strain can be attributed in large part to the favorable effect of deleting glycolaldehyde dehydrogenase AldA which avoids carbon loss occurring through the glycolate/glyoxylate pathway. In addition, our strain does not accumulate synthetic pathway intermediates, as it is the case for xylonate in the fermentation carried out by Liu et al. [9], which positively impacts the productivity of our synthetic pathway. The strain developed by Stephanopoulos et al. [10] which carried the (D)-ribulose-1P pathway produced $42 \mathrm{~g} / \mathrm{L} \mathrm{EG}$ in a fed-batch process at a rate of $0.6 \mathrm{~g} /(\mathrm{L} \mathrm{h})$. No information is available on the actual carbon yield of their fermentation process and on the formation of metabolic by-products. However, in shake flasks this strain reached nearly the same EG yield as our strain (Table 1) which suggests that it should be possible to reach similar 
or even higher yield and productivity through our (D)xylulose-1P synthetic pathway upon further process optimization.

\section{Conclusions}

We have proposed a synthetic pathway for the assimilation of (D)-xylose which relies on the carbon-conserving aldolytic cleavage of the $\mathrm{C} 5$ molecule (D)-xylulose-1P into the $\mathrm{C} 2$ molecule glycolaldehyde and the $\mathrm{C} 3$ molecule DHAP, thus providing an efficient access to the $\mathrm{C} 2 \mathrm{com}$ pounds EG and GA [8]. Spontaneous production of EG upon expression of the synthetic pathway in $E$. coli was shown to depend on the aldehyde reductase YqhD which is induced in the presence of the pathway intermediate glycolaldehyde. The key factors for the optimization of EG production were the deletion of aldA encoding the glycolaldehyde dehydrogenase, and the cultivation of the optimized strains under aerobic conditions. While EG cannot be produced from (D)-xylose via the annotated metabolic network in $E$. coli, we achieved a yield of approximately $0.9 \mathrm{~mol} / \mathrm{mol}$ by applying the new pathway in the genetically optimized production strain. From a conceptual perspective this demonstrates that the stoichiometry of sugar assimilation can be strongly altered such that product formation is favored over cell growth. However, to increase the overall carbon yield of the process, it is required to convert at least part of the DHAP which is released by the synthetic pathway into the desired product. It is therefore of strong interest to test whether the established and suggested metabolic pathways for the conversion of DHAP into GA [25] and EG [10] can be combined with the metabolic pathway developed in our study.

\section{Methods}

\section{Growth media and culture conditions}

Luria-Bertani (LB) medium [26] or M9 minimal medium which contained (D)-glucose or (D)-xylose were used throughout this study. All genetic manipulations were carried out in LB medium. Growth of the cultures was realized in M9 minimal medium that contained (D)glucose or (D)-xylose at concentrations of 20 or $10 \mathrm{~g} / \mathrm{L}$, respectively, together with $18 \mathrm{~g} / \mathrm{L} \mathrm{Na}_{2} \mathrm{HPO}_{4} \cdot 12 \mathrm{H}_{2} \mathrm{O}$, $3 \mathrm{~g} / \mathrm{L} \mathrm{KH}_{2} \mathrm{PO}_{4}, 0.5 \mathrm{~g} / \mathrm{L} \mathrm{NaCl}, 2 / \mathrm{L} g \mathrm{NH}_{4} \mathrm{Cl}, 0.5 \mathrm{~g} / \mathrm{L}$ $\mathrm{MgSO}_{4} \cdot 7 \mathrm{H}_{2} \mathrm{O}, 0.015 \mathrm{~g} / \mathrm{L} \mathrm{CaCl}_{2} \cdot 2 \mathrm{H}_{2} \mathrm{O}, 0.010 \mathrm{~g} / \mathrm{L} \mathrm{FeCl}_{3}$, $0.006 \mathrm{~g} / \mathrm{L}$ Thiamine $\mathrm{HCl}, 0.4 \mathrm{mg} / \mathrm{L}$ NaEDTA $\cdot 2 \mathrm{H}_{2} \mathrm{O}$,

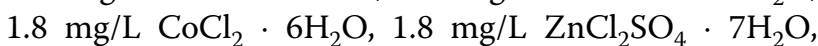
$0.4 \mathrm{mg} / \mathrm{L} \mathrm{Na}_{2} \mathrm{MoO}_{4} \cdot 2 \mathrm{H}_{2} \mathrm{O}, 0.1 \mathrm{mg} / \mathrm{L} \mathrm{H}_{3} \mathrm{BO}_{3}, 1.2 \mathrm{mg} / \mathrm{L}$ $\mathrm{MnSO}_{4} \cdot \mathrm{H}_{2} \mathrm{O}, 1.2 \mathrm{mg} / \mathrm{L} \mathrm{CuCl}{ }_{2} \cdot 2 \mathrm{H}_{2} \mathrm{O} .3$-( $N$-morpholino) propanesulfonic acid (MOPS) solution at $\mathrm{pH} 7$ was used to buffer M9 minimal medium to a final concentration of $20 \mathrm{~g} / \mathrm{L}$ after filter sterilization (Merck Millipore
ExpressPlus). The media were filter sterilized or autoclaved. When required, antibiotics were added to the media at a concentration of 100,50 , and $25 \mu \mathrm{g} / \mathrm{mL}$ for ampicillin, kanamycin and chloramphenicol, respectively. Isopropyl $\beta$-D-1-thiogalactopyranoside (IPTG) was added to a final concentration of $1 \mathrm{mM}$ when needed. All chemicals were from Sigma-Aldrich.

Growth of the cultures was followed by measuring the optical density at $600 \mathrm{~nm}\left(\mathrm{OD}_{600}\right)$ using a spectrophotometer (Biochrom Libra S11). Pre-cultures were grown overnight in $10 \mathrm{~mL}$ of LB medium in $50-\mathrm{mL}$ test tubes (BD Falcon) on a rotary shaker (Infors HT) running at $200 \mathrm{rpm}$ at $37^{\circ} \mathrm{C}$. They were harvested by centrifugation $(4,000 \times g$, Allegra 21-R, Beckman-Coulter), washed once with sterile water and transferred to $250 \mathrm{~mL}$ shake flasks containing M9 minimal medium with glucose adjusting an initial $\mathrm{OD}_{600}$ of 0.25 . IPTG $(1 \mathrm{mM})$ was added to the cultures when the $\mathrm{OD}_{600}$ reached $\sim 0.8$ to assure proper expression of the synthetic pathway enzymes before transfer to xylose medium. After overnight incubation cells were harvested by centrifugation, washed with sterile water and used to inoculate M9 medium containing xylose and $1 \mathrm{mM}$ IPTG. Different aeration conditions were imposed by adding 50 or $100 \mathrm{~mL}$ medium into the $250 \mathrm{~mL}$ shake flasks. Anaerobic conditions were imposed by culturing the cells in $100 \mathrm{~mL}$ medium shaken in $100 \mathrm{~mL}$ culture bottles that were sealed with rubber caps. Absence of oxygen was verified by adding $1 \mathrm{mg} / \mathrm{mL}$ of the redox indicator sodium resazurin (Sigma-Aldrich) to the medium. All cultures were incubated at $37^{\circ} \mathrm{C}$ and shaken at $200 \mathrm{rpm}$ except for the condition with $100 \mathrm{~mL}$ medium in $250 \mathrm{~mL}$ flasks which was shaken at $100 \mathrm{rpm}$ to increase oxygen limitation.

\section{Bioreactor cultures}

The pre-cultures for the inoculation of the bioreactors were cultivated in $500 \mathrm{~mL}$ shake flasks containing $100 \mathrm{~mL}$ xylose mineral medium (composition as indicated above) until exponential phase. The cells were harvested by centrifugation $(4,500 \times g$, Sorvall STR40) and used to inoculate 0.51 bioreactors (MiniBio, Applikon) that contained $250 \mathrm{~mL}$ medium at an $\mathrm{OD}_{600}$ of $\sim 1.2$. The composition of the fermentation medium was similar to the mineral medium used in the shake flask experiments, except that it contained $55 \mathrm{~g} / \mathrm{L}$ (D)-xylose, $2 \mathrm{~g} / \mathrm{L} \mathrm{Na}_{2} \mathrm{HPO}_{4} \cdot 12 \mathrm{H}_{2} \mathrm{O}$, $0.8 \mathrm{~g} / \mathrm{L} \mathrm{KH}_{2} \mathrm{PO}_{4}, 6 \mathrm{~g} / \mathrm{L}\left(\mathrm{NH}_{4}\right)_{2} \mathrm{HPO}_{4}, 0.4 \mathrm{~g} / \mathrm{L}\left(\mathrm{NH}_{4}\right)_{2} \mathrm{SO}_{4}$, $1 \mathrm{~g} / \mathrm{L}$ tryptone (Biokar), $0.5 \mathrm{~g} / \mathrm{L}$ yeast extract (Biokar), $0.4 \mathrm{~mL} / \mathrm{L}$ polypropylene glycol as antifoaming agent, $1 \mathrm{mM}$ IPTG, and no MOPS. The $\mathrm{pH}$ of the cultures was kept at 7.0 by the addition of $10 \mathrm{M} \mathrm{KOH}$, and reactors were aerated with air at $1 \mathrm{vvm}$. Dissolved oxygen tension was regulated by adjusting the appropriate agitation 
Table 4 Escherichia coli strains used in this study

\begin{tabular}{|c|c|c|}
\hline Strain reference & Genotype & References \\
\hline MG1655 & $F^{-} \lambda^{-}$ilvG-rfb-50 rph-1 & ATCC 47076 \\
\hline NEB5- $\alpha$ & fhuA2 $\Delta$ (argF-lacZ) U169 phoA glnV44 $\Phi 80 \Delta$ (lacZ)M15 gyrA96 recA1 relA1 endA1 thi-1 hsdR17 & NEB \\
\hline JW3536-2 & $\mathrm{F}-, \Delta(\operatorname{araD}-a r a B) 567, \Delta$ lacZ4787(::rrnB-3), $\lambda-, \Delta x y \mid B 747:: k a n, r p h-1, \Delta($ rhaD-rhaB) 568, hsdR514 & {$[32]$} \\
\hline JW2978-1 & F-, $\Delta\left(\right.$ araD-araB) 567, $\Delta$ lacZ4787(:.rrnB-3), $\lambda^{-}, \Delta y q h D 783: . k a n, r p h-1, \Delta($ rhaD-rhaB)568, hsdR514 & [32] \\
\hline JW1412-1 & F-, $\Delta\left(\right.$ araD-araB) 567, $\Delta$ lacZ4787(::rrnB-3), $\lambda^{-}, \Delta$ aldA776::kan, rph-1, $\Delta$ (rhaD-rhaB)568, hsdR514 & [32] \\
\hline JW1375-1 & F-, $\Delta(\operatorname{araD}-a r a B) 567, \Delta / a c Z 4787(: . r r n B-3), \lambda^{-}, \Delta / d h A 744: . k a n, r p h-1, \Delta($ rhaD-rhaB) 568, hsdR514 & [32] \\
\hline JW2770-1 & F-, $\Delta\left(\right.$ araD-araB) 567, $\Delta$ lacZ4787(::rrnB-3), $\lambda^{-}, \Delta$ fuc0726::kan, rph-1, $\Delta($ rhaD-rhaB) 568, hsdR514 & [32] \\
\hline JW5556-3 & F-, $\Delta\left(\right.$ araD-araB)567, $\Delta$ lacZ4787(::rrnB-3), $\lambda^{-}, r p h-1, \Delta($ rhaD-rhaB)568, $\Delta$ gldA732::kan, hsdR514 & [32] \\
\hline JW5499-1 & F-, $\Delta(\operatorname{araD}-a r a B) 567, \Delta$ lacZ4787(:.rrnB-3), $\lambda^{-}, \Delta d k g A 784: . k a n, r p h-1, \Delta($ rhaD-rhaB) 568 , hsdR514 & {$[32]$} \\
\hline JW0197-1 & F-, $\Delta\left(\right.$ araD-araB)567, $\Delta d k g B 726:: k a n, \Delta$ lacZ4787(::rrnB-3), $\lambda^{-}, r p h-1, \Delta($ rhaD-rhaB) 568, hsdR514 & {$[32]$} \\
\hline JW2970-1 & F-, $\Delta\left(\right.$ araD-araB) 567, $\Delta$ lacZ4787(::rrnB-3), $\lambda^{-}, \Delta y g h Z 775:: k a n, r p h-1, \Delta($ rhaD-rhaB) 568, hsdR514 & {$[32]$} \\
\hline JW1770-5 & F-, $\Delta\left(\right.$ araD-araB) 567, $\Delta$ lacZ4787(:.rrnB-3), $\lambda^{-}$, yeaE778:.:kan, rph-1, $\Delta($ rhaD-rhaB) 568, hsdR514 & [32] \\
\hline JW0409-1 & F-, $\Delta$ (araD-araB)567, $\Delta$ lacZ4787(::rrnB-3), $\Delta$ yajO778::kan, $\lambda^{-}$, rph-1, $\Delta($ rhaD-rhaB) 568, hsdR514 & {$[32]$} \\
\hline Pen79 & JW2978-1 $\triangle g / d A:: F R T$ & This work \\
\hline Pen99 & Pen79 $\Delta$ fucO::FRT & This work \\
\hline Pen155 & MG1655 $\triangle x y \mid B:: F R T$ & {$[8]$} \\
\hline Pen205 & Pen155 containing pEXT20-khkC-aldoB & {$[8]$} \\
\hline Pen222 & Pen205 containing pACT3-gldA & This work \\
\hline Pen223 & Pen205 containing pACT3-yqhD & This work \\
\hline Pen259 & Pen155 $\Delta y q h D:: F R T$ & This work \\
\hline Pen278 & Pen155 $\triangle$ aldA::FRT & This work \\
\hline Pen325 & Pen278 containing pEXT20-khkC-aldoB & This work \\
\hline Pen332 & Pen325 containing pACT3-gldA & This work \\
\hline Pen333 & Pen325 containing pACT3-yqhD & This work \\
\hline Pen334 & Pen205 $\Delta y q h D:: F R T$ containing pEXT20-khkC-aldoB & This work \\
\hline Pen345 & Pen278 $\Delta g / c D: \because F R T$ & This work \\
\hline Pen361 & Pen345 containing pEXT20-khkC-aldoB & This work \\
\hline Pen641 & Pen325 containing pACT3-fucO & This work \\
\hline Pen644 & Pen205 containing pACT3-fucO & This work \\
\hline Pen877 & MG1655 containing pEXT20-Khk-C-aldoB & This work \\
\hline Pen885 & Pen205 containing empty pACT3 & This work \\
\hline
\end{tabular}

speed (300-1,200 rpm, Rushton rotor, $28 \mathrm{~mm}$ diameter), and was either kept at $40 \%$ to impose fully aerobic conditions, or at $2 \%$ to impose micro-aerobic conditions.

\section{Determination of extracellular metabolite concentrations}

Sugar consumption and production of EG, AG, and other organic acids was followed by regularly withdrawing samples from the supernatant which were centrifuged at $15,700 \mathrm{~g}$ for $5 \mathrm{~min}$ (Eppendorf 5415D), filtered (Sartorius Minisart RC4), and kept at $-20{ }^{\circ} \mathrm{C}$ before being analyzed by HPLC as described previously [8].

RNA extraction, microarray analysis, and data treatment A detailed description of the experimental conditions for RNA extraction and microarray analysis is provided in [8]. Briefly, all experiments were carried on M9 mineral medium containing $10 \mathrm{~g} / \mathrm{L}$ (D)-xylose as the only carbon source. Wild-type cells, wild-type cells exposed to $10 \mathrm{mM}$ glycolaldehyde, and strain Pen205 were harvested during early exponential phase at an $\mathrm{OD}_{600}$ of $\sim 1$. Glycolaldehyde was added to the cultures $30 \mathrm{~min}$ before harvest. Cells were separated from the culture medium by centrifugation $(1,500 \times g, 5 \mathrm{~min}$, Eppendorf $5415 \mathrm{D})$. After discarding the supernatant RNA was extracted from the cells by using the RNeasy Mini Kit (QIAGEN). Quantity and quality of the samples were determined by NanoDrop (Thermo) and Bioanalyzer (Agilent Technologies), respectively. The Low Input Quick Amp Labeling kit (Agilent) was used to convert RNA samples to labeled cDNA which was hybridized on E. coli Gene Expression Microarrays $(8 \times 15 \mathrm{~K}$, Agilent $)$ following the Agilent One-Color Microarray-Based Gene Expression Analysis 
Table 5 Primers used in this study

\begin{tabular}{|c|c|}
\hline Primer & Sequence \\
\hline \multicolumn{2}{|c|}{ Cloning of gldA } \\
\hline gldA_rbs_f & $\begin{array}{l}\text { CCTCTAGAGTCGACCTGCAGAGGAGGATTCATATGGAC- } \\
\text { CGCATTA }\end{array}$ \\
\hline gldA_rbs_r & GCCAAAACAGAAGCTTTTATTCCCACTCTTGCAGG \\
\hline \multicolumn{2}{|c|}{ Cloning of fucO } \\
\hline fucO_rbs_f & $\begin{array}{l}\text { TTGGATCCAGGAGGATTCATATGGCTAACAGAAT- } \\
\text { GATTCT }\end{array}$ \\
\hline fucO_rbs_r & TTAAGCTTTTACCAGGCGGTATGGTAA \\
\hline \multicolumn{2}{|c|}{ Cloning of yqhD } \\
\hline yqhD_rbs_f & $\begin{array}{l}\text { CCTCTAGAGTCGACCTGCAGAGGAGGATTCATAT- } \\
\text { GAACAACTTTA }\end{array}$ \\
\hline yqhD_rbs_r & GCCAAAACAGAAGCTTTTAGCGGGCGGCTTCGT \\
\hline \multicolumn{2}{|c|}{ Verification primers for gene knock-outs } \\
\hline xylB_loc_f & GTTATCGGTAGCGATACCGGGCATTTT \\
\hline xylB_loc_r & GGATCCTGAATTATCCCCCACCCGGTCAGGCA \\
\hline yqhD_loc_f & CGCCATACAACAAACGCACA \\
\hline yqhD_loc_r & CCAGATGCCAGCGGATAACA \\
\hline gldA_loc_f & CGGTTCAGGAGCTGCAAACGCTG \\
\hline gldA_loc_r & TAAGAGTCACAGATTCGACCTTC \\
\hline fucO_loc_f & ACAACATCATGGGCTTATCG \\
\hline KANseq_rev & ATGCGATGTTTCGCTTGGTG \\
\hline aldA_loc_f & TCATCCATGCATGGCAAACG \\
\hline aldA_loc_r & ACTGCCGAAGAGGTGAATAA \\
\hline
\end{tabular}

Restriction sites are italicized and the start codons are shown in bolditalics.

Protocol. The slides were scanned on a Tecan scanner MS200 and analyzed by Feature Extraction V.11.5.1.1. RNA was extracted and analyzed from three independent experiments for each condition. Background correction and normalization of the transcriptome data was carried out as described previously [27-29].

To produce the heatmap shown in Fig. $2 \mathrm{~b}$, the expression levels of the corresponding genes were $\log 2$ transformed, and the genes were clustered according to the Euclidean distance between their expression levels using complete-linkage clustering [30].

\section{Strain and plasmid construction}

(Strain construction) Escherichia coli K-12 substr. MG1655 (ATCC 47076) was used as the parental strain throughout this study. The deletion mutants were created by the phage transduction method adapted from Miller [31]. Briefly: (Lysate preparation) The phage lysates were prepared from strains of the KEIO collection [32] which carried single deletions. The corresponding deletion mutant was grown in $5 \mathrm{~mL}$ of LB medium in $50 \mathrm{~mL}$ test tubes (BD Falcon) at $37^{\circ} \mathrm{C}$ and $200 \mathrm{rpm}$ overnight. The next day, $200 \mu \mathrm{L}$ of this preculture was inoculated into fresh $10 \mathrm{~mL} \mathrm{LB}$ medium supplemented with glucose $(0.2 \%)$ and $\mathrm{CaCl}_{2}(5 \mathrm{mM})$. It was then cultivated for $1 \mathrm{~h}$ at $37^{\circ} \mathrm{C}$ and $200 \mathrm{rpm}$. $100 \mu \mathrm{L}$ of phage lysate P1 was then added into the culture. $200 \mu \mathrm{L}$ chloroform was added to the culture after $2 \mathrm{~h}$ of incubation at $37{ }^{\circ} \mathrm{C}$ and $200 \mathrm{rpm}$. Cells were harvested by centrifugation at 4,500 $\mathrm{g}$ for $10 \mathrm{~min}$ (Allegra 21-R, Beckman-Coulter). The supernatant was transferred to a new tube and $200 \mu \mathrm{L}$ chloroform were added. The lysates were stored at $4{ }^{\circ} \mathrm{C}$ until transduction was carried out. (Transduction) $1.5 \mathrm{~mL}$ of an overnight culture were resuspended in $10 \mathrm{mM}$ $\mathrm{MgSO}_{4}$ and $5 \mathrm{mM} \mathrm{CaCl}{ }_{2} .100 \mu \mathrm{L}$ of this suspension was mixed with $100 \mu \mathrm{L}$ of the lysate and incubated at $30{ }^{\circ} \mathrm{C}$ for $30 \mathrm{~min}$. Then $100 \mu \mathrm{L}$ of $1 \mathrm{M}$ trisodium citrate $\left(\mathrm{Na}_{3} \mathrm{C}_{6} \mathrm{H}_{5} \mathrm{O}_{7}\right)$ solution was added to the cells. They were mixed and $400 \mu \mathrm{L}$ of $\mathrm{LB}$ was added into the cells. They were incubated at $37^{\circ} \mathrm{C}$ and $200 \mathrm{rpm}$ for $1 \mathrm{~h}$. Cells were inoculated on LB agar plates that contained kanamycin. Positive clones were verified by PCR analysis. All strains used and constructed in this study are indicated in Table 4.

(Plasmid construction) Construction of plasmid pEXT20-khkC-aldoB was described previously [8]. The glycolaldehyde-encoding genes gldA, yqhD and fucO were PCR amplified from Escherichia coli K-12 MG1655 genomic DNA using Phusion polymerase (Biolabs) and the corresponding primers listed in Table 5. The PCR

Table 6 Plasmids used in this study

\begin{tabular}{|c|c|c|}
\hline Name & Relevant characteristics & References \\
\hline pGEM-T & Amp ${ }^{R}$, used for PCR fragment subcloning & Promega \\
\hline pACT3 & $\mathrm{Cm}^{\mathrm{R}}$ & {$[33]$} \\
\hline pEXT20 & $A m p^{R}$ & [33] \\
\hline pCP20 & Amp $p^{R}$, plasmid used for removing Kan cassette & [34] \\
\hline pEXT20-khkC-aldoB & pEXT20 derivative carrying both H. sapiens khk-c gene from Asipu et al., 2003 and aldoB gene & {$[8]$} \\
\hline pACT3-gldA & PACT3 derivative carrying gldA gene from E. coli & This work \\
\hline pACT3-yqhD & PACT3 derivative carrying yahD gene from E. coli & This work \\
\hline рАCT3-fucO & PACT3 derivative carrying fucO gene from E. coli & This work \\
\hline
\end{tabular}


products were then purified by using a PCR purification kit (Thermo Scientific). The In-Fusion ${ }^{\circledR}$ HD Cloning Kit (Clontech) was used to recombine the DNA fragments into the pACT3 expression vector that was digested with PstI/HindIII. The vectors obtained were named pACT3gldA, pACT3-yqhD and pACT3-fucO (Table 6).

\section{Additional file}

Additional file 1. Log2 transformed expression levels of candidate glycolaldehyde reductases

\begin{abstract}
Authors' contributions
CeA performed most experiments, interpreted data and wrote the manuscript. YC, DT performed shake flask experiments and interpreted data. CIA performed fermentation experiments. LS, AV performed HPLC analyses. FB and PB analyzed transcriptome data. JMF contributed to the analysis of the data and to the writing of this work. TW conceived the project, oversaw the research, and wrote the manuscript. All authors read and approved the final manuscript.
\end{abstract}

\section{Author details}

1 INSA, UPS, INP, LISBP, Université de Toulouse, 135 Avenue de Rangueil, 31077 Toulouse, France. ${ }^{2}$ UMR792 Ingénierie des Systèmes Biologiques et des Procédés (LISBP), INRA, Toulouse, France. ${ }^{3}$ CNRS, UMR5504, Toulouse, France. ${ }^{4}$ TWB, 3 rue Ariane, 31520 Ramonville-St. Agnes, France. ${ }^{5}$ UMR CNRS 5219, Institut de Mathématiques de Toulouse, INSA, Université de Toulouse, Toulouse, France.

\section{Acknowledgements}

The study was in part financed by the Toulouse White Biotechnology (TWB) consortium (Project: PENTOSYS). DT was supported by a post-doctoral grant (Science without borders program) provided by the CAPES foundation (Ministry of Education, Brazil). YC was supported by a post-doctoral grant provided by the Institut National de la Recherche Agronomique—Region Midi-Pyrénées (INRA, France).

\section{Compliance with ethical guidelines}

\section{Competing interests}

The authors declare that they have no competing interests.

Received: 1 August 2015 Accepted: 4 August 2015

Published online: 04 September 2015

\section{References}

1. Bailey FE, Koleske JV (1976) Poly(ethylene oxide). Academic Press, New York

2. Child J, Willetts A (1978) Microbial metabolism of aliphatic glycols bacterial metabolism of ethylene glycol. Biochimica et Biophysica Acta (BBA) 538(2):316-327

3. Cox DP, Perlman D (1978) The biodegradation of polyethylene glycols. In: Advances in Applied Microbiology, vol 23. Academic Press, pp 173-194

4. Mono-ethylene glycol. http://www.shell.com/global/products-services/ solutions-for-businesses/chemicals/media-centre/factsheets/meg.html. Accessed 15 May 2015

5. Rebsdat S, Mayer D (2000) Ethylene glycol. In: Ullmann's encyclopedia of industrial chemistry. Wiley-VCH Verlag GmbH \& Co. KGaA

6. Curran KA, Alper HS (2012) Expanding the chemical palate of cells by combining systems biology and metabolic engineering. Metab Eng 14(4):289-297

7. Zhang J, Babtie A, Stephanopoulos G (2012) Metabolic engineering enabling technology of a bio-based economy. Curr Opin Chem Eng 1(4):355-362
8. Cam Y, Alkim C, Trichez D, Trebosc V, Vax A, Bartolo F et al (2015) Engineering of a synthetic metabolic pathway for the assimilation of (D)-xylose into value-added chemicals. ACS Synth Biol. doi:10.1021/ acssynbio.5b00103

9. Liu H, Ramos K, Valdehuesa KN, Nisola G, Lee W-K, Chung W-J (2013) Biosynthesis of ethylene glycol in Escherichia coli. Appl Microbiol Biotechnol 97(8):3409-3417

10. Stephanopoulos G, Pereira B, De MM, Dugar D, Avalos JL (2013) Engineering microbes and metabolic pathways for the production of ethylene glycol. Patent; WO2013126721 A1

11. Lee C, Kim I, Park C (2013) Glyoxal detoxification in Escherichia coli K-12 by NADPH dependent aldo-keto reductases. J Microbiol 51(4):527-530

12. Cocks GT, Aguilar J, Lin ECC (1974) Evolution of I-1,2-propanediol catabolism in Escherichia coli by recruitment of enzymes for L-fucose and L-lactate metabolism. J Bacteriol 118(1):83-88

13. Boronat A, Aguilar J (1979) Rhamnose-induced propanediol oxidoreductase in Escherichia coli: purification, properties, and comparison with the fucose-induced enzyme. J Bacteriol 140(2):320-326

14. Chen YM, Lin EC (1984) Dual control of a common L-1,2-propanediol oxidoreductase by L-fucose and L-rhamnose in Escherichia coli. J Bacteriol 157(3):828-832

15. Tang CT, Ruch FE, Lin CC (1979) Purification and properties of a nicotinamide adenine dinucleotide-linked dehydrogenase that serves an Escherichia coli mutant for glycerol catabolism. J Bacteriol 140(1):182-187

16. Obradors N, Cabiscol E, Aguilar J, Ros J (1998) Site-directed mutagenesis studies of the metal-binding center of the iron-dependent propanediol oxidoreductase from Escherichia coli. Eur J Biochem 258(1):207-213

17. Subedi KP, Kim I, Kim J, Min B, Park C (2008) Role of GldA in dihydroxyacetone and methylglyoxal metabolism of Escherichia coli K12. FEMS Microbiol Lett 279(2):180-187

18. Jarboe $L$ (2011) YahD: a broad-substrate range aldehyde reductase with various applications in production of biorenewable fuels and chemicals. Appl Microbiol Biotechnol 89(2):249-257

19. Turner $P$, Miller $E$, Jarboe L, Baggett $C$, Shanmugam KT, Ingram L (2011) YqhC regulates transcription of the adjacent Escherichia coli genes yqhD and $\mathrm{dkgA}$ that are involved in furfural tolerance. J Ind Microbiol Biotechnol 38(3):431-439

20. Baldomà L, Aguilar J (1987) Involvement of lactaldehyde dehydrogenase in several metabolic pathways of Escherichia coli K12. J Biol Chem 262(29):13991-13996

21. Pellicer MT, Badia J, Aguilar J, Baldomà L (1996) glc locus of Escherichia coli: characterization of genes encoding the subunits of glycolate oxidase and the glc regulator protein. J Bacteriol 178(7):2051-2059

22. Lord JM (1972) Glycolate oxidoreductase in Escherichia coli. Biochim Biophys Acta (BBA) Bioenerg 267(2):227-237

23. Walther T, Hensirisak P, Agblevor FA (2001) The influence of aeration and hemicellulosic sugars on xylitol production by Candida tropicalis. Bioresour Technol 76:213-220

24. Twigg RS (1945) Oxidation-reduction aspects of resazurin. Nature 155(3935):401-402

25. Dischert W, Soucaille P (2012) Method for producing high amount of glycolic acid by fermentation. Patent W010108909 A1

26. Bertani G (1951) Studies on lysogenesis. I. The mode of phage liberation by lysogenic Escherichia coli. J Bacteriol 62(3):293-300

27. Ritchie ME, Silver J, Oshlack A, Holmes M, Diyagama D, Holloway A et al (2007) A comparison of background correction methods for two-colour microarrays. Bioinformatics 23:2700-2707

28. Bolstad BM, Irizarry RA, Astrand M, Speed TP (2003) A comparison of normalization methods for high density oligonucleotide array data based on variance and bias. Bioinformatics 19:185-193

29. Yang YH, Thorne NP (2003) Normalization for two-color cDNA microarray data. In: Statistics and science: a Festschrift for Terry Speed. Institute of Mathematical Statistics, Beachwood, OH, pp 403-418. doi:10.1214/ Inms/1215091155

30. Eisen MB, Spellman PT, Brown PO, Botstein D (1998) Cluster analysis and display of genome-wide expression patterns. Proc Natl Acad Sci 95(25):14863-14868

31. Miller J (1992) A short course in bacterial genetics: a laboratory manual and handbook for Escherichia coli and related bacteria, Cold Spring Harbor Laboratory Press, Plainview, NY 
32. Baba T, Ara T, Hasegawa M, Takai Y, Okumura Y, Baba M et al (2006) Construction of Escherichia coli K-12 in-frame, single-gene knockout mutants: the Keio collection. Mol Syst Biol 2(1):1-11

33. Dykxhoorn DM, St Pierre R, Linn T (1996) A set of compatible tac promoter expression vectors. Gene 177(1-2):133-136
34. Cherepanov PP, Wackernagel W (1995) Gene disruption in Escherichia coli: TcR and KmR cassettes with the option of Flp-catalyzed excision of the antibiotic-resistance determinant. Gene 158(1):9-14
Submit your next manuscript to BioMed Central and take full advantage of:

- Convenient online submission

- Thorough peer review

- No space constraints or color figure charges

- Immediate publication on acceptance

- Inclusion in PubMed, CAS, Scopus and Google Scholar

- Research which is freely available for redistribution

Submit your manuscript at www.biomedcentral.com/submit 